\title{
Update on the challenges of treating trigeminal neuralgia
}

This article was published in the following Dove Press journal:

Orphan Drugs: Research and Reviews

10 April 2015

Number of times this article has been viewed

\section{Mark Obermann}

Department of Neurology, University of Duisburg-Essen, Essen, Germany

Correspondence: Mark Obermann Department of Neurology, University of Duisburg-Essen, Hufelandstrasse 55, Essen 45I22, Germany

Tel +49 20I 72384385

Fax +4920I 7235542

Email mark.obermann@uni-due.de
Abstract: Despite the multitude of treatment options currently available for trigeminal neuralgia, its management remains challenging in a considerable number of patients. The response to any particular treatment can be quite variable interindividually, and personalized treatment options are both resource-consuming and time-consuming. Anticonvulsant drugs, muscle relaxants, and neuroleptic agents are the preferred medical treatment for trigeminal neuralgia. Large placebocontrolled clinical trials are scarce, and no specific established substance has been developed for the treatment of trigeminal neuralgia. Promising new treatment options currently in clinical evaluation are botulinum neurotoxin type A injections and CNV1014802, a novel sodium channel blocker that selectively blocks the Nav1.7 sodium channel. Patients who do not respond to medical therapy may be eligible for more invasive treatment options, such as percutaneous Gasserian ganglion techniques, gamma knife surgery, and microvascular decompression.

Keywords: trigeminal neuralgia, treatment, current, future, options, orphan drugs

\section{Introduction}

Trigeminal neuralgia (TN) is defined by the International Headache Society as a "unilateral disorder characterized by brief electric shock-like pains, abrupt in onset and termination, and limited to the distribution of one or more divisions of the trigeminal nerve". ${ }^{1}$ The new International Classification of Headache Disorder-III beta recommends that classical TN (essential or idiopathic) be classified into TN with and without concomitant persistent facial pain. ${ }^{1,2}$ Secondary forms caused by tumor, trauma, multiple sclerosis, or post-herpetic neuralgia exist, and are classified by International Classification of Headache Disorders-III beta as secondary painful trigeminal neuropathies. According to the new classification, classical TN should now be diagnosed even if a slight hyperesthesia or hypoesthesia is present in the individual patient. ${ }^{2} \mathrm{TN}$ typically starts in the second or third divisions of the trigeminal nerve. ${ }^{1}$ The ophthalmic nerve is involved in less than $5 \%$ of cases, and may be associated with other differential diagnoses such as trigeminal autonomic cephalalgias. ${ }^{3}$ A characteristic TN attack usually lasts for less than 1 second to a few seconds. However, it can occur in clusters of varying intensity and duration with up to 2 minutes. In many patients, the attack is followed by a brief refractory period during which a new stimulation is not able to evoke another attack. ${ }^{4}$ The patient is usually pain-free between paroxysms, but sometimes a dull, concomitant background pain may persist. ${ }^{1}$ The pathophysiological mechanisms underlying this persistent pain remain unknown, but it has been shown that a poor medical and surgical outcome is associated with concomitant background pain in TN. ${ }^{5-8}$ 
This review summarizes the existing knowledge concerning the customary treatment options for TN on the basis of recent reports from the Quality Standards Subcommittee of the American Academy of Neurology ${ }^{9}$ and the European Federation of Neurological Societies. ${ }^{10}$ Moreover, a MEDLINE search (on December 30, 2014) for publications containing the term "trigeminal neuralgia" was performed. We limited the search to the previous 3 years. It returned 629 publications, of which 83 were review articles. An additional Google search was performed to find press releases about more recent, experimental, and pilot studies concerning treatment of TN.

\section{Disease burden}

Pain from TN imposes an extensive burden on patients. During the most severe attacks, affected patients may be unable to eat or speak. Some patients fear that the pain could return suddenly at any time even between attacks. ${ }^{11}$ This results in serious impairment of daily functioning as well as quality of life. Reduced measures of quality of life, daily functioning, well-being, mood, sleep, and overall health status were correlated with pain severity. ${ }^{12}$ Employment was impacted in $34 \%$ of TN patients. Moderate to severe pain within the previous 24 hours was reported by up to two-thirds of patients, and depression is quite frequent in this patient population. ${ }^{13-15}$

\section{Diagnostics}

The most important factor for adequate treatment is a correct clinical diagnosis. Patient history is the most essential diagnostic tool. Trigeminal autonomic cephalalgias, eg, cluster headache, SUNCT (Short-lasting Unilateral Neuralgiform headache attacks with Conjunctival injection and Tearing), and paroxysmal hemicrania are important to differentiate, and patients with pain only in the first division are particularly suspicious for the presence of a trigeminal autonomic cephalalgia. ${ }^{16}$ The current diagnostic criteria need reconfirmation by clinical practice. ${ }^{17,18}$ Special diagnostic procedures primarily aim at differentiation of symptomatic TN from classical TN. ${ }^{9,10}$ Structural causes can be detected by routine head imaging in up to $15 \%$ (95\% confidence interval [CI] 11-20) of patients, not including neurovascular conflict. Cerebellopontine angle tumors (ie, acoustic neuroma/vestibular schwannoma, meningioma, cerebellar astrocytoma) and multiple sclerosis plaques are the most commonly identified abnormalities. Trigeminal reflex testing, including the blink reflex, has a relatively high accuracy for identifying patients with symptomatic TN. It has a pooled specificity of $87 \%$
(95\% CI 77-93) and a pooled sensitivity of 94\% (95\% CI 91-97) in five evaluated studies. On the other hand, evoked potentials were unable to sufficiently distinguish classical TN from symptomatic TN (pooled specificity 64\% [95\% CI 56-71], pooled sensitivity 84\% [95\% CI 73-92]). ${ }^{9,10}$

Imaging plays a major role in determining the presence of neurovascular conflict, especially in the presurgical assessment. Specificities and sensitivities can vary (specificity $29 \%-93 \%$, sensitivity $52 \%-100 \%$ ), which is probably related to different magnetic resonance imaging sequences being used in different investigations. ${ }^{9,10}$ Therefore, the usefulness of magnetic resonance imaging in determining vascular contact remains uncertain. A very recent large imaging study that included 135 patients with TN revealed that neurovascular conflict was present on the symptomatic side as well as the asymptomatic side ( $89 \%$ versus $78 \%$, odds ratio $2.4,95 \%$ CI 1.2-4.8, $P=0.017)$. However, severe neurovascular conflict presents much more often on the symptomatic side than on the asymptomatic side (53\% versus $13 \%$, odds ratio $11.6,95 \% \mathrm{CI} 4.7-28.9, P<0.001)$. In one study, arteries were found to cause severe neurovascular contact in $98 \%$ of cases. ${ }^{19}$ This study showed nicely that neurovascular contact causing displacement or atrophy of the trigeminal nerve is in fact strongly associated with the symptomatic side in classical $\mathrm{TN}$ as opposed to neurovascular contact in general.

\section{Established medical treatment}

Surgical and pharmacological treatment strategies are numerous, widely used, and often effective. Medical therapy should be started first, with surgical interventions considered in patients only after two failed treatment attempts. There are no studies that directly compare surgical and medical treatment. Active participation in a support group can aid patients cope to better with their disease and remain compliant with medical therapy. ${ }^{20}$

\section{First-line treatment}

Carbamazepine 200-1,200 mg/day should be first-line treatment. Alternatively, oxcarbazepine $600-1,800 \mathrm{mg} /$ day can be used, as suggested by current therapy guidelines. ${ }^{9,10}$ There is stronger evidence for carbamazepine, ${ }^{21-24}$ but the safety profile of oxcarbazepine is much better. ${ }^{25}$ Its mechanism of analgesic action most likely relates to blockade of voltage-sensitive sodium channels, which leads to stabilization of hyperexcited cell membranes, reduction of propagation of synaptic impulses, and/or inhibition of repetitive firing. Generally, a smaller dose is required in the early treatment of TN to be effective, and much less than what 
would be required for the treatment of epilepsy. Sometimes the pain responds to as little as $100 \mathrm{mg}$ three times or even twice per day. The daily dosage should be increased reasonably rapidly by $100 \mathrm{mg}$ every other day for the remaining patients until pain control can be established or side effects become intolerable. Generally, 300-800 mg/day divided into two to three daily doses is considered to be effective. Approximately $80 \%$ of patients benefit initially, but higher doses are often required over time in order to maintain efficacy. Autoinduction of carbamazepine leads to a decline in efficacy in about $50 \%$ of patients..$^{21}$ Frequent side effects are nausea, drowsiness, diplopia, dizziness, ataxia, hyponatremia, and elevation of transaminases. Allergic rash, hepatotoxicity, myelosuppression, systemic lupus erythematosus, StevensJohnson syndrome, lymphadenopathy, and aplastic anemia are potentially serious but uncommon side effects. The US Food and Drug Administration recommends genetic testing for patients of Asian descent, because they are genetically at the greatest risk of developing Stevens-Johnson syndrome. Serum sodium measurements, liver function tests, and a complete blood count need to be performed after 2-4 weeks of treatment in order to detect complications early on. The ketoanalog of carbamazepine is oxcarbazepine, which is rapidly converted into the pharmacologically active 10-monohydroxy metabolite. The metabolite has only a mild effect on hepatic enzyme induction and thus has a much improved side effect profile. ${ }^{26}$ Oxcarbazepine is an alternative to carbamazepine and is usually started at $150 \mathrm{mg}$ twice daily, and increased by $300 \mathrm{mg}$ every 3 days until pain relief is achieved without intolerable side effects. Generally, 300-600 mg twice daily is the required maintenance dose.

\section{Second-line treatment}

There is limited evidence supporting second-line treatment recommendations. Commonly used treatments are lamotrigine $400 \mathrm{mg} /$ day ${ }^{27}$ baclofen $40-80 \mathrm{mg} /$ day, ${ }^{28}$ or pimozide 4-12 mg/day. Pimozide has potential long-term side effects, such as extrapyramidal symptoms, so is seldom used in clinical practice. Baclofen, a $\mathrm{GABA}_{\mathrm{B}}$ receptor agonist, decreases excitatory neurotransmission. Double-blind studies have demonstrated its efficacy in up to $70 \%$ of patients at doses of 10-60 mg daily. ${ }^{28}$ However, in a 5-year follow-up study of 60 patients, efficacy was maintained in only $30 \%$ of cases, while $17 \%$ reported recurrence of pain within 3-6 months and $22 \%$ reported loss of response within 18 months. ${ }^{28}$ Common side effects are drowsiness, lassitude, gastrointestinal discomfort, and dizziness. Baclofen has the second best scientific evidence supporting its efficacy after carbamazepine.
Lamotrigine blocks voltage-sensitive sodium channels, inhibits the release of excitatory neurotransmitters, and stabilizes neural membranes. In a randomized, controlled trial, lamotrigine showed superiority to placebo in 14 patients with TN refractory to carbamazepine. ${ }^{27}$ The starting dose is $25 \mathrm{mg} /$ day, and should be increased slowly to $200-400 \mathrm{mg}$ daily. Side effects include nausea, dizziness, ataxia, and blurred vision. Skin rash can occur in approximately 7\%-10\% of patients within the first $4-8$ weeks of treatment. ${ }^{29}$ Desquamation and severe rash associated with symptoms of fever and lymphadenopathy are signs of Stevens-Johnson syndrome, which requires rapid discontinuation of the drug. These side effects are less likely to occur when the titration is done very slowly. Unfortunately, many patients cannot tolerate slow and cautious titration due to pain. ${ }^{30}$

\section{Alternative treatment options}

Other antiepileptic drugs have been investigated in small controlled or open-label trials. Phenytoin, gabapentin, clonazepam, topiramate, pregabalin, valproate, and levetiracetam, as well as tocainide $12 \mathrm{mg} /$ day, have shown some benefit. ${ }^{31}$ Newer antiepileptic drugs are particularly promising for future investigation as they usually have fewer drug interactions and less severe side effects. The incidence of TN increases with age, ${ }^{32}$ so age-related physiological changes that may alter pharmacokinetics, eg, reduced renal and hepatic function, less predictable drug protein-binding, decreased blood flow, and interactions with drugs used to treat concomitant disorders, will become more and more relevant for patient safety and treatment efficacy. Carbamazepine cannot be tolerated by approximately 6\%-10\% of patients. ${ }^{33}$ Its use is further limited by a narrow therapeutic window as well as multiple pharmacological interactions. Lamotrigine, gabapentin, pregabalin, levetiracetam, and topiramate are promising in this regard because they have very few drug interactions. In a study of 36 patients, gabapentin alone or in combination with a local injection of ropivacaine demonstrated adequate efficacy. ${ }^{34}$ Gabapentin is started at $300 \mathrm{mg}$ per day and can be increased slowly by $300 \mathrm{mg}$ every 2-3 days if tolerated. Relatively minor side effects and a lack of drug interactions are the main features of gabapentin. Side effects include somnolence, dizziness, diarrhea, headache, nausea, confusion, and ankle swelling. An open-label study of gabapentin was performed in 53 patients with TN (14 with constant concomitant facial pain) with follow-up over 1 year, and the drug proved effective at a dose of 150-600 mg per day. TN pain was reduced in over $50 \%$ of cases, with largely sustained efficacy in $74 \%$ of 
patients during follow-up. Patients with concomitant facial pain had a worse outcome $(7 / 14,50 \%, P=0.02)$ than those without persistent concomitant facial pain $(32 / 39,82 \%){ }^{6}$ Topiramate $100-400 \mathrm{mg} /$ day showed efficacy in $75 \%$ of a small sample of eight patients. ${ }^{35}$ Levetiracetam was tested over a 10-week period in an open-label prospective pilot study that included ten patients with TN. A dose of up to $4,000 \mathrm{mg}$ per day was administered, and $40 \%$ of patients $(n=4)$ reported an improvement of pain 50\%-90\%. ${ }^{36}$ These preliminary findings need to be confirmed by randomized controlled trials in the future.

Tizanidine, a centrally acting alpha-adrenergic agonist, showed efficacy in a small, double-blind, crossover study in $80 \%$ of ten patients with TN. However, after $1-3$ months of follow-up, all patients experienced recurrence of pain. ${ }^{37,38}$

Neuralgia crisis is often treated with phenytoin, which has proven to be effective in a small uncontrolled study. Pain relief was achieved by an intravenous dose of $14 \mathrm{mg} / \mathrm{kg}$, which lasted for 1-2 days. This is approximately the time frame during which oral medication such as carbamazepine starts to work. ${ }^{39}$ Lidocaine $8 \%$ administered as a nasal spray also achieved temporary relief of neuropathic pain, at least in the second division. ${ }^{40}$ Sumatriptan $3 \mathrm{mg}$ administered subcutaneously achieved marked analgesia in $80 \%$ of patients in a double-blind, placebo-controlled study of 24 patients with otherwise refractory $\mathrm{TN}$. The median duration of pain relief was 8 hours. ${ }^{41}$ Local opioid analgesia of the superior cervical ganglion is an alternative treatment strategy, and was evaluated retrospectively in 74 patients with neuropathic facial pain. Seventy-three percent of patients reported clinically relevant pain reduction, with a pain reduction of $\geq 50 \%$ in $59 \%$ of patients with the first treatment. ${ }^{42}$

\section{Surgical treatment}

Patients who are refractory to medical treatment comprising at least two adequately dosed drugs including carbamazepine should be considered for surgical intervention. This decision should be based on the patient's symptoms and not on neuroimaging results. ${ }^{43}$

Percutaneous Gasserian ganglion procedures, gamma knife surgery, and microvascular decompression are efficacious and the generally recommended interventional treatment options. Reconfirmation of efficacy meeting evidence-based medicine standards is warranted. Surgical therapy for $\mathrm{TN}$ is either destructive, with the sensory function of the trigeminal nerve being destroyed intentionally, or nondestructive, with decompression of the trigeminal nerve and preservation of its normal functioning. Percutaneous
Gasserian ganglion techniques include radiofrequency and balloon compression, percutaneous glycerol rhizolysis, and thermocoagulation.

Unfortunately, due to the descriptive nature of most of these studies, informed evidence-based decision-making regarding surgical treatment remains difficult, and the best time to consider surgical intervention is as yet unclear. ${ }^{44}$ Some TN experts recommend early surgical referral for patients not adequately responding to first-line medical therapy. Others suggest trying at least two different drugs alone and in combination before proceeding to surgical intervention. There is no clear evidence supporting either of these recommendations, so treatment decisions need to be made on a case-by-case basis.

\section{Novel upcoming medical treatment options}

Botulinum neurotoxin type A (BoNT-A) has shown some efficacy in the treatment of TN in recent studies. Its proposed mechanism involves local release of antinociceptive neuropeptides, such as glutamate, substance P, and calcitonin gene-related peptide, thereby reducing peripheral and central sensitization. ${ }^{45}$ A small uncontrolled clinical trial $(n=13)$ showed significant symptom relief following treatment with BoNT-A. BoNT-A was administered directly into the painful area of the face at a mean subcutaneous dose of $3.22 \mathrm{U} / \mathrm{cm}^{2}$. After 60 days, the therapeutic effect of BoNT-A had gradually faded. ${ }^{46}$ A current randomized, placebo-controlled, doubleblind study has investigated BoNT-A $25 \mathrm{U}$ or $75 \mathrm{U}$ (placebo $\mathrm{n}=28)$, BTX-A $25 \mathrm{U}(\mathrm{n}=27)$, and BoNT-A $75 \mathrm{U}(\mathrm{n}=29)$ in 84 patients with classical TN. The duration of the study was 8 weeks for each patient. Endpoints were efficacy, pain severity, and adverse reactions. The BTX-A $25 \mathrm{U}$ and $75 \mathrm{U}$ groups showed a significant reduction of pain on a visual analog scale compared with placebo after 1 week, and remained stable throughout the study. Response rates in the $25 \mathrm{U}(70.4 \%)$ and $75 \mathrm{U}(86.2 \%)$ groups were significantly higher than in the placebo group (32.1\%) at week 8 , and there was no significant difference in response rate between the $25 \mathrm{U}$ and $75 \mathrm{U}$ groups. Using the Patient Global Impression of Change scale, $66.7 \%$ of the $25 \mathrm{U}$ group and $75.9 \%$ of the $75 \mathrm{U}$ group reported that their pain symptoms were "much improved" or "very much improved" versus $32.1 \%$ of the placebo group. All adverse reactions were graded as mild or moderate. ${ }^{47}$ Further controlled clinical trials are warranted to reconfirm the efficacy of botulinum toxin for the treatment of TN.

A promising new substance has recently completed Phase II clinical investigation, with positive results. 
CNV1014802 is a novel, small-molecule, state-dependent sodium channel blocker that exhibits potency and selectivity against the Nav1.7 sodium channel. The study used a novel randomized withdrawal design to demonstrate its efficacy. ${ }^{48}$ Following an initial 21-day, open-label treatment period with CNV1014802 $150 \mathrm{mg}$ three times a day, patients who showed a successful response in the final week were randomized to a 28-day, double-blind treatment period with either CNV1014802 $150 \mathrm{mg}$ or placebo three times a day. Response was defined as a $30 \%$ or more reduction in number or severity of paroxysms relative to the run-in period. Sixty-seven patients were recruited into the study with $69 \%$ completed the open-label period and entered the double-blind phase.

CNV1014802 was well tolerated and showed a consistent reduction of pain severity and number of paroxysms in all primary and secondary outcomes. In terms of the primary endpoint of the study, there was a treatment failure rate of $33 \%$ for CNV1014802 versus $65 \%$ for placebo and a favorable separation from placebo on the Kaplan-Meier time to relapse. CNV1014802 achieved a 2.3-unit decrease on the Numeric Rating Scale for pain intensity, a $60 \%$ reduction in paroxysms versus $12 \%$ for placebo, and pain severity decreased by $55 \%$ versus $18 \%$ on placebo. There were no serious drug-related adverse events, and the adverse event profile of the drug was similar to that of placebo in the double-blind phase of the study. ${ }^{49}$ However, even though these results are promising, the evaluation period was short and this potential treatment option will have to demonstrate its efficacy in the long term.

\section{Neuromodulation techniques}

Repetitive transcranial magnetic stimulation is an emerging technology that introduces the possibility of assessing whether patients with trigeminal neuropathic pain will respond to direct epidural cortical stimulation by first measuring their response to a trial of noninvasive cortical stimulation. In a study of $24 \mathrm{TN}$ patients given repetitive transcranial magnetic stimulation to the motor cortex at $20 \mathrm{~Hz}$ daily for 5 days, pain ratings decreased by approximately $45 \%$ for 2 weeks. ${ }^{50} \mathrm{In}$ another study of 12 patients with chronic intractable TN who had failed surgical treatment, $58 \%$ experienced a greater than $30 \%$ reduction in pain after receiving repetitive transcranial magnetic stimulation. ${ }^{51}$

Patient self-administered transcranial direct current stimulation of the motor cortex had a good effect on pain reduction in patients with classical $\mathrm{TN}$. Ten patients were stimulated daily for 20 minutes over 2 weeks using anodal $(1 \mathrm{~mA})$ or sham transcranial direct current stimulation over the primary motor cortex (M1) in a randomized double-blind crossover design. The primary outcome variable was pain intensity on a $0-10$ verbal rating scale. Anodal transcranial direct current stimulation reduced pain intensity by $29 \%$ after 2 weeks of treatment $(P=0.0008)$. The attack frequency was within the same effect range, but did not reach statistical significance. No severe adverse events were reported. Anodal transcranial direct current stimulation over 2 weeks ameliorates the intensity of pain in patients with TN. It may become a valuable treatment option for patients who do not respond to conventional treatment.

\section{Conclusion}

Among the many treatment options available for management of TN, very few have proven efficacy according to modern evidence-based medicine standards. Treatment of patients who suffer from TN remains challenging, because the interindividual response rate to different treatments can be quite variable. New treatment options are just starting to emerge, and promising orphan drugs are being clinically tested on larger patient populations exclusively for this rare but very disabling disease for the first time. This continuous effort by researchers, the pharmaceutical industry, and clinicians may lead to more efficient, more specific, and better tolerated treatment options for patients with TN in the future.

\section{Disclosure}

The author has received scientific support, travel support, and/or honoraria from Biogen Idec, Novartis, Sanofi-Aventis, Genzyme, Pfizer, Teva, and Heel. He has also received research grants from Allergan, Electrocore, Heel, and the German Ministry for Education and Research.

\section{References}

1. International Headache Society. The International Classification of Headache Disorders: 2nd Edition. Cephalalgia. 2004;24 Suppl 1: 9-160.

2. Headache Classification Committee of the International Headache Society (IHS). The International Classification of Headache Disorders, 3rd edition (beta version). Cephalalgia. 2013;33(9):629-808.

3. De Simone R, Marano E, Brescia Morra V, et al. A clinical comparison of trigeminal neuralgic pain in patients with and without underlying multiple sclerosis. Neurol Sci. 2005;26 Suppl 2:s150-s151.

4. Maarbjerg S, Gozalov A, Olesen J, Bendtsen L. Trigeminal neuralgia - a prospective systematic study of clinical characteristics in 158 patients. Headache. 2014;54(10):1574-1582.

5. Szapiro J Jr, Sindou M, Szapiro J. Prognostic factors in microvascular decompression for trigeminal neuralgia. Neurosurgery. 1985;17(6): 920-929.

6. Obermann M, Yoon MS, Sensen K, Maschke M, Diener HC, Katsarava Z. Efficacy of pregabalin in the treatment of trigeminal neuralgia. Cephalalgia. 2008;28(2):174-181. 
7. Sandell T, Eide PK. Effect of microvascular decompression in trigeminal neuralgia patients with or without constant pain. Neurosurgery. 2008;63(1):93-99.

8. Hagenacker T, Bude V, Naegel S, et al. Patient-conducted anodal transcranial direct current stimulation of the motor cortex alleviates pain in trigeminal neuralgia. J Headache Pain. 2014;15:78.

9. Gronseth G, Cruccu G, Alksne J, et al. Practice parameter: the diagnostic evaluation and treatment of trigeminal neuralgia (an evidence-based review): report of the Quality Standards Subcommittee of the American Academy of Neurology and the European Federation of Neurological Societies. Neurology. 2008;71(15):1183-1190.

10. Cruccu G, Gronseth G, Alksne J, et al. AAN-EFNS guidelines on trigeminal neuralgia management. Eur J Neurol. 2008;15(10):1013-1028.

11. Cheshire WP. Trigeminal neuralgia feigns the terrorist. Cephalalgia. 2003;23(3):230.

12. Tolle T, Dukes E, Sadosky A. Patient burden of trigeminal neuralgia: results from a cross-sectional survey of health state impairment and treatment patterns in six European countries. Pain Pract. 2006;6(3): 153-160.

13. Marbach JJ, Lund P. Depression, anhedonia and anxiety in temporomandibular joint and other facial pain syndromes. Pain. 1981;11(1): 73-84.

14. Zakrzewska JM, Jassim S, Bulman JS. A prospective, longitudinal study on patients with trigeminal neuralgia who underwent radiofrequency thermocoagulation of the Gasserian ganglion. Pain. 1999;79:51-58.

15. Zakrzewska JM. Insights: Facts and Stories Behind Trigeminal Neuralgia. Gainesville, FL, USA: Trigeminal Neuralgia Association; 2006.

16. Cohen AS, Matharu MS, Goadsby PJ. Short-lasting unilateral neuralgiform headache attacks with conjunctival injection and tearing (SUNCT) or cranial autonomic features (SUNA) - a prospective clinical study of SUNCT and SUNA. Brain. 2006;129 Pt 10:2746-2760.

17. Zebenholzer K, Wober C, Vigl M, Wessely P, Wober-Bingol C. Facial pain and the second edition of the International Classification of Headache Disorders. Headache. 2006;46(2):259-263.

18. Zakrzewska JM. Diagnosis and differential diagnosis of trigeminal neuralgia. Clin J Pain. 2002;18(1):14-21.

19. Maarbjerg S, Wolfram F, Gozalov A, Olesen J, Bendtsen L. Significance of neurovascular contact in classical trigeminal neuralgia. Brain. 2015;138 Pt 2:311-319.

20. Zakrzewska JM, Jorns TP, Spatz A. Patient led conferences - who attends, are their expectations met and do they vary in three different countries? Eur J Pain. 2009;13(5):486-491.

21. Campbell FG, Graham JG, Zilkha KJ. Clinical trial of carbazepine (Tegretol) in trigeminal neuralgia. J Neurol Neurosurg Psychiatry. 1966;29(3):265-267.

22. Killian JM, Fromm GH. Carbamazepine in the treatment of neuralgia. Use of side effects. Arch Neurol. 1968;19(2):129-136.

23. Nicol CF. A four year double-blind study of tegretol in facial pain. Headache. 1969;9(1):54-57.

24. Rockliff BW, Davis EH. Controlled sequential trials of carbamazepine in trigeminal neuralgia. Arch Neurol. 1966;15(2):129-136.

25. Beydoun A. Safety and efficacy of oxcarbazepine: results of randomized, double-blind trials. Pharmacotherapy. 2000;20(8 Pt 2):152S-158S

26. Martinez W, Ingenito A, Blakeslee M, Barkley GL, McCague K, D'Souza J. Efficacy, safety, and tolerability of oxcarbazepine monotherapy. Epilepsy Behav. 2006;9(3):448-456.

27. Zakrzewska JM, Chaudhry Z, Nurmikko TJ, Patton DW, Mullens EL. Lamotrigine (Lamictal) in refractory trigeminal neuralgia: results from a double-blind placebo controlled crossover trial. Pain. 1997;73: 223-230.

28. Fromm GH, Terrence CF, Chattha AS. Baclofen in the treatment of trigeminal neuralgia: double-blind study and long-term follow-up. Ann Neurol. 1984;15:240-244.

29. Wiffen PJ, Rees J. Lamotrigine for acute and chronic pain. Cochrane Database Syst Rev. 2007;2:CD006044.

30. Ketter TA, Wang PW, Chandler RA, et al. Dermatology precautions and slower titration yield low incidence of lamotrigine treatment-emergent rash. J Clin Psychiatry. 2005;66(5):642-645.
31. Lindstrom $P$, Lindblom $U$. The analgesic effect of tocainide in trigeminal neuralgia. Pain. 1987;28(1):45-50

32. Khan OA. Gabapentin relieves trigeminal neuralgia in multiple sclerosis patients. Neurology. 1998;51(2):611-614

33. Taylor JC, Brauer S, Espir ML. Long-term treatment of trigeminal neuralgia with carbamazepine. Postgrad Med J. 1981;57(663):16-18.

34. Lemos L, Flores S, Oliveira P, Almeida A. Gabapentin supplemented with ropivacaine block of trigger points improves pain control and quality of life in trigeminal neuralgia patients when compared with gabapentin alone. Clin J Pain. 2008;24(1):64-75.

35. Domingues RB, Kuster GW, Aquino CC. Treatment of trigeminal neuralgia with low doses of topiramate. Arq Neuropsiquiatr. 2007;65(3B): 792-794.

36. Jorns TP, Johnston A, Zakrzewska JM. Pilot study to evaluate the efficacy and tolerability of levetiracetam (Keppra) in treatment of patients with trigeminal neuralgia. Eur J Neurol. 2009;16(6): 740-744.

37. Fromm GH, Aumentado D, Terrence CF. A clinical and experimental investigation of the effects of tizanidine in trigeminal neuralgia. Pain. 1993;53(3):265-271.

38. Vilming ST, Lyberg T, Lataste X. Tizanidine in the management of trigeminal neuralgia. Cephalalgia. 1986;6(3):181-182.

39. Cheshire WP. Fosphenytoin: an intravenous option for the management of acute trigeminal neuralgia crisis. J Pain Symptom Manage. 2001; 21(6):506-510

40. Kanai A, Suzuki A, Kobayashi M, Hoka S. Intranasal lidocaine $8 \%$ spray for second-division trigeminal neuralgia. Br J Anaesth. 2006;97(4): 559-563.

41. Kanai A, Saito M, Hoka S. Subcutaneous sumatriptan for refractory trigeminal neuralgia. Headache. 2006;46(4):577-582.

42. Elsner F, Radbruch L, Gaertner J, Straub U, Sabatowski R. [Efficacy of opioid analgesia at the superior cervical ganglion in neuropathic head and facial pain]. Schmerz. 2006;20(4):268-272. German.

43. Cheshire WP. Can MRI distinguish injurious from innocuous trigeminal neurovascular contact? J Neurol Neurosurg Psychiatry. 2005;76(11): 1470-1471.

44. Spatz AL, Zakrzewska JM, Kay EJ. Decision analysis of medical and surgical treatments for trigeminal neuralgia: how patient evaluations of benefits and risks affect the utility of treatment decisions. Pain. 2007;131(3):302-310.

45. Aoki KR. Review of a proposed mechanism for the antinociceptive action of botulinum toxin type A. Neurotoxicology. 2005;26(5):785-793.

46. Piovesan EJ, Teive HG, Kowacs PA, Della Coletta MV, Werneck LC, Silberstein SD. An open study of botulinum-A toxin treatment of trigeminal neuralgia. Neurology. 2005;65(8):1306-1308.

47. Zhang H, Lian Y, Ma Y, et al. Two doses of botulinum toxin type A for the treatment of trigeminal neuralgia: observation of therapeutic effect from a randomized, double-blind, placebo-controlled trial. J Headache Pain. 2014;15:65.

48. Zakrzewska JM, Palmer J, Ettlin DA, et al. Novel design for a phase IIa placebo-controlled, double-blind randomized withdrawal study to evaluate the safety and efficacy of CNV1014802 in patients with trigeminal neuralgia. Trials. 2013;14:402.

49. Convergence Pharmaceuticals. Convergence Pharmaceuticals' Novel Sodium Channel Blocker CNV1014802 Shows Excellent Efficacy and Safety in Ground-Breaking Phase II Trigeminal Neuralgia Study [press release], 2014. Available from www.convergencepharma.com/userfiles/ file/140616_Convergence_TGN\%20vFINALFINAL.pdf. Accessed December 30, 2014

50. Khedr EM, Kotb H, Kamel NF, Ahmed MA, Sadek R, Rothwell JC. Long-lasting antalgic effects of daily sessions of repetitive transcranial magnetic stimulation in central and peripheral neuropathic pain. J Neurol Neurosurg Psychiatry. 2005;76(6):833-838.

51. Lefaucheur JP, Drouot X, Menard-Lefaucheur I, et al. Neurogenic pain relief by repetitive transcranial magnetic cortical stimulation depends on the origin and the site of pain. J Neurol Neurosurg Psychiatry. 2004;75(4):612-616. 


\section{Publish your work in this journal}

Orphan Drugs: Research and Reviews is an international, peer-reviewed, open access journal publishing original research, reports, reviews and commentaries on all areas of the design and development of orphan drugs for the treatment of rare diseases through to clinical applications. Clinical outcomes, patient safety, and programs for the development and

Submit your manuscript here: http://www.dovepress.com/orphan-drugs-research-and-reviews-journ effective, safe, and sustained use of medicines will be a feature of the journal. The manuscript management system is completely online and includes a very quick and fair peer-review system, which is all easy to use. Visit http://www.dovepress.com/testimonials.php to read real quotes from published authors. 